\title{
Members of the aquaporin family in the developing pea seed coat include representatives of the PIP, TIP, and NIP subfamilies
}

\author{
Jolanda A.M.J. Schuurmans ${ }^{1,3,+}$, Joost T. van Dongen ${ }^{1,4,+}$, Bas P.W. Rutjens ${ }^{1,3}$, Alex \\ Boonman $^{1,5}$, Corné M.J. Pieterse ${ }^{2}$ and Adrianus C. Borstlap ${ }^{1, *}$ \\ ${ }^{1}$ Transport Physiology and ${ }^{2}$ Section of Phytopathology, Utrecht University, Sorbonnelaan 16, 3584 CA Utrecht, \\ Netherlands (*author for correspondence; e-mail a.c.borstlap @bio.uu.nl); present addresses: ${ }^{3}$ Molecular Plant \\ Physiology, Utrecht University, Padualaan 8, 3584 CH Utrecht, Netherlands; ${ }^{4}$ Max Planck Institute of Molecular \\ Plant Physiology, Am Mühlenberg 1,14476 Golm, Germany; ${ }^{5}$ Plant Ecophysiology Group, Utrecht University, \\ Sorbonnelaan 16, 3584 CA Utrecht, Netherlands; ${ }^{+}$these authors contributed equally to the work
}

Received 6 June 2003; accepted in revised form 27 October 2003

Key words: aquaglyceroporins, aquaporins, MIPs, seed coat, seed development, seed germination

\begin{abstract}
Water and nutrients required by developing seeds are mainly supplied by the phloem and have to be released from a maternal parenchyma tissue before being utilized by the filial tissues of embryo and endosperm. To identify aquaporins that could be involved in this process four full-length cDNAs were cloned and sequenced from a cDNA library of developing seed coats of pea (Pisum sativum L.). The cDNA of PsPIP1-1 appeared to be identical to that of clone 7a/TRG-31, a turgor-responsive gene cloned previously from pea roots. PSPIP1-1, PsPIP2-1, and PSTIP11 , or their possible close homologues, were also expressed in cotyledons of developing and germinating seeds, and in roots and shoots of seedlings, but transcripts of PSNIP- 1 were only detected in the seed coat. In mature dry seeds, high hybridization signals were observed with the probe for PsPIP1-1, but transcripts of PSPIP2-1, PSTIP1-1, and PSNIP-1 were not detected. Functional characterization after heterologous expression in Xenopus oocytes showed that PsPIP2-1 and PsTIP1-1 are aquaporins whereas PsNIP-1 is an aquaglyceroporin. PsNIP-1, like several other NIPs, contains a tryptophan residue corresponding with Trp- 48 in GlpF (the glycerol facilitator of Escherichia coli) that borders the selectivity filter in the permeation channel. It is suggested that PsPIP1-1 and/or its possible close homologues could play a role in water absorption during seed imbibition, and that PsPIP2-1, possibly together with PsPIP1-1, could be involved in the release of phloem water from the seed coat symplast, which is intimately connected with the release of nutrients for the embryo.
\end{abstract}

Abbreviations: MIPs, major intrinsic proteins; NIPs, nodulin 26-like intrinsic proteins; PIPs, plasma membrane intrinsic proteins; SIPs, small, basic intrinsic proteins; TIPs, tonoplast intrinsic proteins

\section{Introduction}

The phloem is the food-conducting tissue of vascular plants. Its sieve elements constitute a symplasmic route by which organic nutrients (mainly sucrose and amino acids) along with inorganic nutrients (mainly $\mathrm{K}^{+}$) are translocated hydraulically from source to sink (Münch, 1930; Fisher, 2000). Developing seeds are strong sinks, particularly during the storage phase. In developing legume seeds the nutrients arriving by the phloem are transferred, via plasmodesmata, from the sieve elements to adjacent parenchyma cells of the seed coat (phloem unloading). Because the embryonic tissues are symplasmically isolated from the maternal seed coat, the nutrients have to be released from seed coat parenchyma cells into the apoplast (seed coat unloading). The molecular basis for the efflux of these solutes is still unknown. It has been suggested that in seed coats of common bean (Phaseolus vulgaris) and broad bean (Vicia faba) sucrose is 
released by an $\mathrm{H}^{+}$-antiport mechanism (Patrick and Offler, 2001), whereas work in our group on pea has provided evidence for poorly selective pores in the plasma membrane of seed coat parenchyma cells that allow the passage of sugars, amino acids and possibly inorganic ions as well (de Jong et al., 1996, 1997; van Dongen et al., 2001).

Seed coat unloading implies the transport of a range of nutrients as well as of phloem water (Münch water) across the cell membranes of seed coat parenchyma cells. It may be anticipated that aquaporins are involved in the efflux of water from the parenchyma cells. Aquaporins belong to the family of major intrinsic proteins (MIPs), an ancient family with members in all life forms (Agre et al., 1998; van Dongen and Borstlap, 2003). Based on sequence homology, members of the MIP family in higher plants fall into four major subgroups: plasma membrane intrinsic proteins (PIPs), tonoplast intrinsic proteins (TIPs), Nodulin 26-like MIPs (NLMs or NIPs), and small, basic intrinsic proteins (SIPs). Representatives of the PIPs, TIPs, NIPs, and SIPs have been identified in the moss Physcomitrella patens, indicating that the four subfamilies were already established when land plant evolution began (Borstlap, 2002). The PIP subfamily can be further divided into the highly homologous but clearly distinct subgroups PIP1 and PIP2, whereas the TIP subfamily consists of five subgroups, including the $\alpha-, \gamma-$, and $\delta$-TIPs which may be markers of different types of vacuoles (Jauh et al., 1999). According to a new nomenclature the subgroups of $\alpha-, \gamma-$, and $\delta$-TIPs are now designated TIP3, TIP1, and TIP2, respectively (Johanson et al., 2001; Chaumont et al., 2001).

Functionally, MIPs fall into two main categories: aquaporins and glycerol facilitators (Heymann and Engel, 1999; Agre et al., 1998). High-resolution atomic structures of prototypes of each category, aquaporin-1 (AQP1) from mammals and the glycerol facilitator (GlpF) from Escherichia coli, have elucidated aspects of the selectivity of these transporters ( $\mathrm{Fu}$ et al., 2000; Sui et al., 2001). To some extent the glycerol-transporting MIPs, including GlpF, also allow the passage of water (Borgnia and Agre, 2001), hence their designation as aquaglyceroporins. Some mammalian MIPs transport larger molecules or ions. AQP9 from the liver allows the passage of water and a variety of neutral molecules including carbamides, polyols, purines and pyrimidines (Tsukaguchi et al., 1998), whereas AQP6, an endomembrane aquaporin in renal epithelia, is permeable to anions, particularly nitrate (Ikeda et al., 2002).

Seed coat parenchyma cells are committed to release a range of solutes that serve as nutrients for the developing seed tissues and as raw materials for the synthesis of storage compounds. Münch's mass flow concept of phloem transport dictates that this solute release must be intimately connected with the release of water. The question may be raised whether MIPs are involved in the flux of water and solutes across the membrane of seed coat parenchyma cells. Here we report the cloning, sequencing and functional characterization of four MIP genes from a cDNA library of developing pea seed coats, which turned out to encode members of the PIP1, PIP2, TIP1, and NIP subgroup, respectively.

\section{Materials and methods}

\section{Plant material}

Pea plants (Pisum sativum L. cv. Marzia) were grown in a growth chamber as described (de Jong and Wolswinkel, 1995). Developing seeds used for RNA gel blot analysis were taken from pods tagged at the time of flowering. To obtain seedling tissues for RNA gel blot analysis, pea seeds were imbibed in aerated tap water for $24 \mathrm{~h}$ and then placed on moist filter paper in petri dishes in the dark at room temperature.

\section{Construction of a seed coat cDNA library}

Coats from developing pea seeds in the filling stage were collected and immediately frozen in liquid nitrogen. Total RNA was isolated from $10 \mathrm{~g}$ fresh weight of seed coats by the guanidine hydrochloride extraction method (Logemann et al., 1987). Poly(A) ${ }^{+}$RNA was prepared by oligo(dT)-cellulose chromatography (Sambrook et al., 1989). The library was constructed in the $\lambda$ Uni-ZAP XR vector and packaged into phage particles with the ZAP-cDNA Gigapack II Gold Cloning kit from Stratagene. The resulting library consisted of $9.7 \times 10^{6}$ plaque forming units with an average insert length of $1.1-1.3 \mathrm{~kb}$.

\section{Reverse transcriptase polymerase chain reaction $(R T-P C R)$}

PCR was performed with two degenerate primers corresponding to the conserved NPA boxes of the MIP family. The sense primer $5^{\prime}-(\mathrm{T} / \mathrm{C}) \mathrm{T}(\mathrm{A} / \mathrm{C} / \mathrm{G} / \mathrm{T}) \mathrm{AA}(\mathrm{C} / \mathrm{T})$ 
CC(A/C/G/T)GC(A/C/G/T)GT(A/C/G/T)AC-3' corresponds to the amino acid sequence Leu-Asn-ProAla-Val-Thr in the B-loop, and the antisense primer $5^{\prime}$ $\mathrm{AA}(\mathrm{A} / \mathrm{C} / \mathrm{G} / \mathrm{T})(\mathrm{C} / \mathrm{G})(\mathrm{A} / \mathrm{T})(\mathrm{A} / \mathrm{C} / \mathrm{G} / \mathrm{T}) \mathrm{C}(\mathrm{G} / \mathrm{T})(\mathrm{A} / \mathrm{C} / \mathrm{G} / \mathrm{T})$ GC(A/C/G/T)GG(A/G)TT-3' corresponds to the sequence Asn-Pro-Ala-Arg-Ser-Phe in the E-loop. Firststrand cDNA was synthesized from $5 \mu \mathrm{g}$ of poly(A) ${ }^{+}$ RNA from pea seed coats with M-MuLV reverse transcriptase (Boehringer) and the antisense primer. The PCR-mixture consisted of $10 \mathrm{mM}$ Tris- $\mathrm{HCl} \mathrm{pH} 9.0$, $50 \mathrm{mM} \mathrm{KCl}, 1.5 \mathrm{mM} \mathrm{MgCl} 2,200 \mu \mathrm{M}$ of each deoxynucleotide triphosphate, $3.8 \mu \mathrm{M}$ of each primer, cDNA corresponding to $300 \mathrm{ng}$ of poly(A) ${ }^{+} \mathrm{RNA}$, and 1.5 units of $T a q$ DNA polymerase (Boehringer) in a total volume of $50 \mu \mathrm{l}$. After 4 min of denaturation at $92{ }^{\circ} \mathrm{C}$ the amplification was performed in 35 cycles $\left(1 \min 92^{\circ} \mathrm{C}, 1 \min 50{ }^{\circ} \mathrm{C}\right.$, and $1 \min 72^{\circ} \mathrm{C}$ ). PCR products were subjected to phenol/chloroform extractions and precipitated with ethanol. Primers were removed on a SephacrylS-400HR column (Pharmacia). After electrophoresis on an agarose gel a band corresponding to a ca. $400 \mathrm{bp} \mathrm{PCR} \mathrm{product} \mathrm{was} \mathrm{isol-}$ ated, purified with QiaexII (Qiagen) and cloned into the pGEM-3Z plasmid vector (Promega).

\section{Labeling of cDNA probes and screening of the cDNA library}

The PCR fragments of PSPIP1-1, PsPIP2-1, PsTIP11 and $P S N I P-1$ were radiolabeled with $\left[\alpha{ }^{32} \mathrm{P}\right] \mathrm{dCTP}$ $(3000 \mathrm{Ci} / \mathrm{mmol}$; ICN) by random primer labeling (Feinberg and Vogelstein, 1983) with 40-50 ng of cDNA as a template (Ready-to-Go-DNA-labeling kit, Pharmacia). Excess nucleotides were removed with Microspin S400-HR columns (Pharmacia). The cDNA library was plated on the Escherichia coli host strain XL1-Blue MRF'. Plaques were blotted onto Hybond$\mathrm{N}^{+}$membranes (Amersham) and hybridization was performed for $16-18 \mathrm{~h}$ at $63{ }^{\circ} \mathrm{C}$ in $0.5 \mathrm{M}$ sodium phosphate $\mathrm{pH} 7.2,1 \mathrm{mM}$ EDTA and 7\% w/v SDS, with the $\left[{ }^{32} \mathrm{P}\right]$-labeled probes of the four MIP gene fragments. Blots were washed three times for $30 \mathrm{~min}$ at $65{ }^{\circ} \mathrm{C}$ in $2 \times \mathrm{SSC}, 0.5 \% \mathrm{w} / \mathrm{v}$ SDS and autoradiographed (Kodak X-Omat AR) at $-80^{\circ} \mathrm{C}$ for $2-5$ days. Positive clones were isolated from the agar plates and stored in phage buffer $(20 \mathrm{mM}$ Tris- $\mathrm{HCl} \mathrm{pH}$ 7.4, $100 \mathrm{mM} \mathrm{NaCl}, 10 \mathrm{mM} \mathrm{MgSO}$, $0.01 \%$ gelatin). A second screening was performed similarly except that the phage suspension was plated at lower density, allowing the isolation of single phage clones. Following Stratagene's protocol of the ZAP-cDNA Gigapack II
Gold Cloning kit, a helper phage (Exassist) was added to the clones selected from the second screening to excise in vivo the pBluescript phagemid from the $\lambda$ vector.

\section{RNA gel blot analysis}

Collected plant material was frozen immediately in liquid nitrogen and ground to a fine powder using a mortar and pestle. Aliquots of the homogenized tissue $(0.5 \mathrm{~g})$ were stored at $-80^{\circ} \mathrm{C}$ until use for RNA extraction. Total RNA was extracted in an equal volume of extraction buffer $(0.35 \mathrm{M}$ glycine, $0.048 \mathrm{M} \mathrm{NaOH}$, $0.34 \mathrm{M} \mathrm{NaCl}, 0.04 \mathrm{M}$ EDTA, 4\% w/v SDS). The homogenates were extracted with phenol and chloroform and the RNA was precipitated with $\mathrm{LiCl}$ as described by Sambrook et al. (1989). For RNA analysis, $15 \mu \mathrm{g}$ of RNA was denatured with glyoxal and DMSO (Sambrook et al., 1989), separated electrophoretically on $1.5 \% \mathrm{w} / \mathrm{v}$ agarose gels and blotted onto Hybond- $\mathrm{N}^{+}$membranes by capillary transfer. The electrophoresis buffer and blotting buffer consisted of $10 \mathrm{mM}$ and $25 \mathrm{mM}$ sodium phosphate $\mathrm{pH} 7$, respectively. Prior to hybridization RNA blots were washed in $20 \mathrm{mM}$ Tris- $\mathrm{HCl} \mathrm{pH} 8.0$ at $65{ }^{\circ} \mathrm{C}$ for $1 \mathrm{~h}$. RNA blots were hybridized overnight at $65{ }^{\circ} \mathrm{C}$ in $0.5 \mathrm{M}$ sodium phosphate $\mathrm{pH} 7.2,7 \% \mathrm{w} / \mathrm{v}$ SDS, $1 \mathrm{mM}$ EDTA with the $\left[{ }^{32} \mathrm{P}\right]$-labeled, ca. 400 bp PCR fragments of PSPIP1-1, PSPIP2-1, PSTIP1-1 and $P S N I P-1$ as probes. Blots were washed three times for $30 \mathrm{~min}$ at $65{ }^{\circ} \mathrm{C}$ with $2 \times \mathrm{SSC}, 0.5 \%$ w/v SDS and hybridization signals were detected by autoradiography $\left(-80^{\circ} \mathrm{C}\right.$; Kodak X-Omat-AR) or by phosphoimaging (BioRad Molecular Imager FX; program Quantity One). Film scans and phosphoimager files were processed in Adobe PhotoShop 5.5. Hybridization with an 851 bp RT-PCR fragment (sense primer 5'-GGCTCATTGAGTCTGGTA-3', antisense primer 5'-GTCGAGGTCTCGTTCGTT-3') from Pisum sativum 18S rRNA (accession number U43011) was used as loading control.

\section{Sequencing and sequence analysis}

Sequencing was performed with the DyeDeoxy Terminator Cycle Sequencing kit. Similarity searches were performed by the Advanced BLAST2 Search Service of Bork group at EMBL, Heidelberg (Altschul et al., 1997). Multiple alignments were performed by MultAlin, University of Toulouse, France (Corpet, 1988). 
Subcloning into an oocyte expression vector

cDNA clones encoding PsPIP1-1, PsPIP2-1, PsTIP11 and PsNIP-1 were inserted in pT7TS, a vector for transient expression in Xenopus oocytes (Johnson and Krieg, UT, Austin, TX). This vector, which is closely related to pSP64T (Krieg and Melton, 1984), contains a $5^{\prime}$ - and $3^{\prime}$-untranslated region that originates from Xenopus $\beta$-globin flanking the polylinker site and a $3^{\prime}$ poly(A) tail of 30 nucleotides to increase cRNA stability after injection in Xenopus oocytes. Sticky ends of the cDNA clones were made blunt using the Klenow fragment of DNA polymerase I (USB, Cleveland, USA) and ligated into the EcoRV site of pT7TS. Constructs were sequenced to ensure that no errors were introduced in the coding sequence. The pT7TS oocyte expression vectors with the different inserts of pea MIP clones were used as template for in vitro cRNA preparation.

\section{Preparation of cRNA}

Enzymes and supplements were obtained from Roche Diagnostics (Mannheim, Germany) unless stated otherwise. Plasmids were isolated with a Plasmid DNA isolation kit (Qiagen, Hilden, Germany) and linearized with SalI. For in vitro transcription, ca. $2 \mu \mathrm{g}$ DNA was incubated for $2 \mathrm{~h}$ at $37^{\circ} \mathrm{C}$ in $50 \mu \mathrm{l}$ transcription mixture (15 U T7 RNA polymerase; Promega, Madison, WI), $1 \mathrm{mM}$ of each rNTP, $0.75 \mathrm{mM}$ of the methylated cap analogue $\left(\mathrm{m}^{7} \mathrm{G}\left(5^{\prime}\right) \mathrm{ppp}\left(5^{\prime}\right) \mathrm{G}\right), 40 \mathrm{U}$ RNasin (Promega), $10 \mathrm{mM}$ DTT, $2 \mathrm{mM}$ spermidine, $10 \mathrm{mM} \mathrm{NaCl}$, $6 \mathrm{mM} \mathrm{MgCl} 2,40 \mathrm{mM}$ Tris, $\mathrm{pH} 7.5$ in ultrapure water). To increase the yield, another $15 \mathrm{U}$ of RNA polymerase was added after $1 \mathrm{~h}$. The reaction was stopped by a 15 min incubation with $2 \mathrm{U}$ of RQ1 RNase-free DNase (Promega) at $37^{\circ} \mathrm{C}$. Proteins were extracted from the reaction mixture by a phenol/chloroform extraction and the free nucleotides were separated from the cRNA using a Sephadex column. G50-Sephadex (fine, DNA grade, Pharmacia, Uppsala, Sweden) in TNE (20 mM Tris pH 7.5, $50 \mathrm{mM} \mathrm{NaCl}, 1$ mM EDTA) was autoclaved and brought into a Pasteur pipette with a small piece of glass wool in the tip $(1.5 \mathrm{ml}$ settled bed volume). The cRNA samples were diluted with sterile ultrapure water to $100 \mu \mathrm{l}$, loaded onto the column, and eluted with TNE. The eluate was collected in fractions of $100 \mu \mathrm{l}$ in which the cRNA concentration was determined spectrophotometrically (GeneQuant, Pharmacia). Intact cRNA was eluted as a distinct peak in fractions 8-11. The cRNA of these fractions was pre- cipitated and dissolved in water to a concentration of $0.5 \mu \mathrm{g} / \mu \mathrm{l}$.

\section{Injection of cRNA into oocytes}

Ovaries were surgically isolated from Xenopus laevis frogs anaesthetized with $0.1 \%$ w/v 3-aminobenzoic acid ethyl ester methanesulfonate salt in water (Sigma Aldrich, Saint Louis, MO) and placed in modified Barth's solution (MBS: $0.33 \mathrm{mM} \mathrm{Ca}\left(\mathrm{NO}_{3}\right)_{2}, 0.41 \mathrm{mM}$ $\mathrm{CaCl}_{2}, 88 \mathrm{mM} \mathrm{NaCl}, 1 \mathrm{mM} \mathrm{KCl}, 2.4 \mathrm{mM} \mathrm{NaHCO}$, $10 \mathrm{mM}$ Hepes $\mathrm{pH} 7.4,0.82 \mathrm{mM} \mathrm{MgSO} 4)$. An ovary was torn into small pieces, containing about 10 oocytes each and incubated in MBS with ca. $15 \mathrm{U}$ collagenase A (Roche Diagnostics) in a final volume of $30 \mathrm{ml}$ for $2 \mathrm{~h}$ at room temperature. At the end of the reaction, the collagenase was carefully washed away by rinsing the oocytes five times in $30 \mathrm{ml}$ MBS. Stage V and VI oocytes (Dumont, 1972) were collected and stored at $18^{\circ} \mathrm{C}$ in MBS supplied with $10 \mu \mathrm{g} / \mathrm{ml}$ gentamycin (Sigma Aldrich). The next day, oocytes were injected with $25 \mathrm{ng}$ cRNA in $50 \mathrm{nl}$ ultrapure water for determining water permeability, or $11.5 \mathrm{ng}$ cRNA in $23 \mathrm{nl}$ ultrapure water for solute uptake experiments by using a Nanoject Auto/Oocyte Injector (Drummond Sci. Co., PA). As a control treatment, oocytes were injected with equal volumes of ultrapure water. The day after injection the vitelline membrane around vital oocytes was manually peeled off with two forceps. This horrid job was made easier by incubating oocytes for $5 \mathrm{~min}$ in a hypertonic solution (250 mM sucrose, $60 \mathrm{mM} \mathrm{KCl}, 10 \mathrm{mM}$ EGTA, $8 \mathrm{mM}$ $\mathrm{MgCl}_{2}, 40 \mathrm{mM}$ Hepes, $\mathrm{pH}$ 7.0) by which the vitelline layer became detached from the oocyte (Silberberg and Magleby, 1997).

\section{Water permeability assay}

The osmotic water permeability of the oocyte membrane was determined one day after cRNA injection. Single oocytes were transferred to $10 \% \mathrm{v} / \mathrm{v}$ MBS and immediately observed under a microscope (Zeiss Axioskoop equipped with a Nikon digital camera DXM1200). For $2 \mathrm{~min}$, a sequence of digital photographs was made at $5 \mathrm{~s}$ intervals. The osmotic water permeability $\left(P_{\mathrm{f}}\right)$ of the oocyte membrane was calculated from the initial rate of cell volume change with the formula

$$
P_{\mathrm{f}}=\frac{V_{0}\left(d V_{\text {rel }} / d t\right)}{S \cdot V_{w} \cdot\left(\text { osm }_{\text {in }}-o s m_{\text {out }}\right)}
$$


AQP1 PsPIP1-1

AQP1

PSPIP1-1

PsPIP2-1

PsץTIP-1

PsNIP-1

GlpF

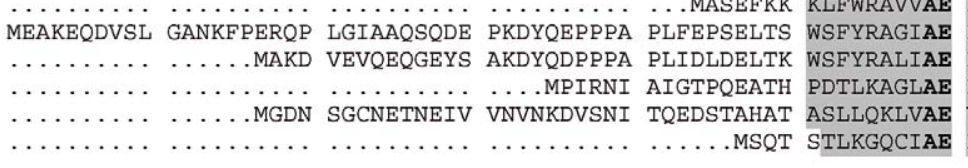

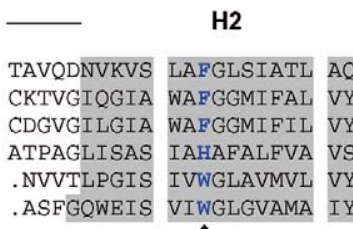

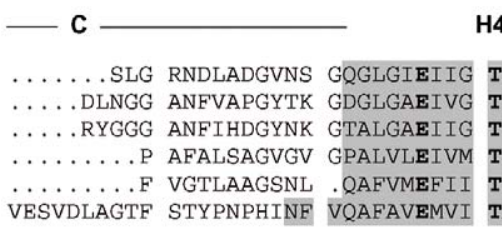

H3
$-\mathbf{E}$

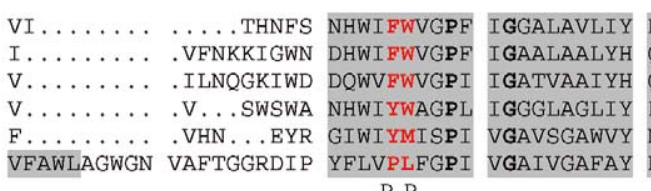

$\mathrm{P}_{4} \mathrm{P}_{5}$
AQP1 PsPIP1-1

PsPIP2-1 Ps $\gamma$ TIP-1 PsNIP-1 GIpF
H4 D D D

IGSALGFKY PVG.... NNQ IATFLFLYI TVLTVMGVVR ES.......SK IATLLFLY TILTIIGYSH OTDANAGGTD ISTFIFVFA GSGSGIAYNK LTD. . . . NGA GTYFLIFA GC.AAVAVNK NND....... GC.AAVAVNK NND $\ldots \ldots$

Figure 1. Alignments of AQP1, GlpF, and the deduced amino acid sequences of the four MIPs cloned from a cDNA library of pea seed coats. PsPIP1-1 is identical to clone 7a/TRG-31 (Guerrero et al., 1990; Guerrero and Crossland, 1993). Transmembrane helices H1-H6 and pore helices B and E (in gray) in the four MIPs from pea were predicted after alignment to AQP1. Residues predicted to border the selectivity filter (blue) are marked by arrows. $\mathrm{P}_{1}-\mathrm{P}_{5}$ denote Froger's positions (red) which have been used to discriminate between aquaporins and glycerol facilitators (Froger et al., 1998).

with initial oocyte volume $V_{0}=0.9 \mathrm{~mm}^{3}$, initial oocyte surface area $S=4.5 \mathrm{~mm}^{2}$, molar volume of water $V_{w}=18 \mathrm{~cm}^{3} / \mathrm{mol}$, the osmolarity in the oocyte $\operatorname{osm}_{\text {in }}=200 \mu \mathrm{mol} / \mathrm{cm}^{3}$ and the osmolarity of the hypotonic medium osm out $=20 \mu \mathrm{mol} / \mathrm{cm}^{3}$.

\section{Solute permeability assay}

To determine solute permeability of the oolemma, uptake experiments with radioactively labeled solutes were performed with 18-24 oocytes per experiment. Oocytes were incubated for $15 \mathrm{~min}$ at room temperature in $3 \mathrm{ml} \mathrm{90 \%} \mathrm{v/v} \mathrm{MBS} \mathrm{supplied} \mathrm{with} \mathrm{ca.} 37 \mathrm{kBq}$ of either $\left[{ }^{14} \mathrm{C}\right]$-glycerol $(5.92 \mathrm{GBq} / \mathrm{mmol}),\left[{ }^{14} \mathrm{C}\right]$-urea (2.11 GBq/mmol), $\left[{ }^{14} \mathrm{C}\right]$-glycine $(3.74 \mathrm{GBq} / \mathrm{mmol})$ or $18.5-37 \mathrm{kBq}\left[{ }^{33} \mathrm{P}\right]$-orthophosphate (ICN, Costa Mesa, USA). In the experiments with phosphate, unlabelled sodium orthophosphate was added to a final concentration of $10 \mu \mathrm{M}$. The $\left[{ }^{14} \mathrm{C}\right]$-labeled chemicals were obtained from Amersham Pharmacia Biotech, Buckinghamshire, UK. After incubation, the oocytes were rinsed three times in ice-cold $90 \%$ v/v MBS. Oocytes were then transferred individually to the wells of a sample plate (Wallac Oy, Turku, Finland) and destructed by incubating in $100 \mu \mathrm{l} 10 \% \mathrm{w} / \mathrm{v}$ SDS at $35{ }^{\circ} \mathrm{C}$ for several hours. To each well $300 \mu 1$ scintillation solution (Optiphase Supermix, Wallac Oy) was added and the amount of radioactivity taken up was measured for every single oocyte by liquid scintillation counting (1450 MicroBeta Plus, Wallac Oy).

\section{Accession numbers}

PSPIPI-1 is identical to clone 7a /TRG-31 (accession number Z18288; Guerrero et al., 1990; Guerrero and Crossland, 1993). Accession numbers for the other nucleotide sequences are as follows: PIP1-2 frag- 
ment, AJ548795; PsPIP2-1, AJ243307; PsTIP1-1, AJ243309; PsNIP-1, AJ243308.

\section{Results}

\section{Cloning and sequencing of four MIPs}

RT-PCR of mRNA from developing pea seed coats was performed with primers corresponding to the conserved NPA boxes of the MIP family. Electrophoresis of the PCR products on agarose gel yielded a single band of ca. $400 \mathrm{bp}$ (not shown). The PCR products were cloned into the plasmid vector pGEM3Z. Transformation of $E$. coli cells yielded 37 independent clones. Five distinct PCR fragments could be identified that showed homology to MIP genes. Two fragments belonged to the PIP1 group, three other fragments to the PIP2, TIP1, and NIP subgroup, respectively. Restriction analysis indicated that 12 clones contained the PIP1-1 fragment, 3 clones the PIP1-2 fragment, 4 clones the PIP2 fragment, 1 clone the TIP1 fragment, and 2 clones the NIP fragment.

To obtain full-length sequences the seed coat cDNA library was screened using the $\left[{ }^{32} \mathrm{P}\right]$-labeled PCR-fragments. From ca. 50000 plaques, ca. 200 positive clones were obtained with the PIPI-1 fragment, 80 with the PIP2 fragment, 80 with the TIP1 fragment, and 40 with the NIP fragment. These abundances of the four transcripts in the cDNA library are in reasonable agreement with their abundances in the mixture of PCR products. Sequencing of the fulllength cDNAs confirmed that they encode members of the PIP1, PIP2, TIP1, and NIP subgroup. The nucleotide sequence of the full-length clone of PSPIP1-1 appeared to be identical to that of clone $7 a / T R G-31$, a turgor-responsive gene cloned previously from pea roots (Guerrero et al., 1990; Guerrero and Crossland, 1993). No attempt was made to obtain a full-length clone of PIP1-2. The other full-length clones were named PSPIP2-1, PSTIP1-1 and PsNIP-1 respectively. An alignment of the deduced amino acid sequences of the four genes together with those of AQP1 and GlpF is shown in Figure 1.

Expression in developing, mature, and germinating seeds

Expression of aquaporin genes was examined by RNA gel blot analysis with the $\left.{ }^{32} \mathrm{P}\right]$-labeled PCR fragments (ca. 400 nucleotides) of PSPIP1-1, PSPIP2-1, PSTIP1-1 and PSNIP-1 as probes. Dot-blots showed

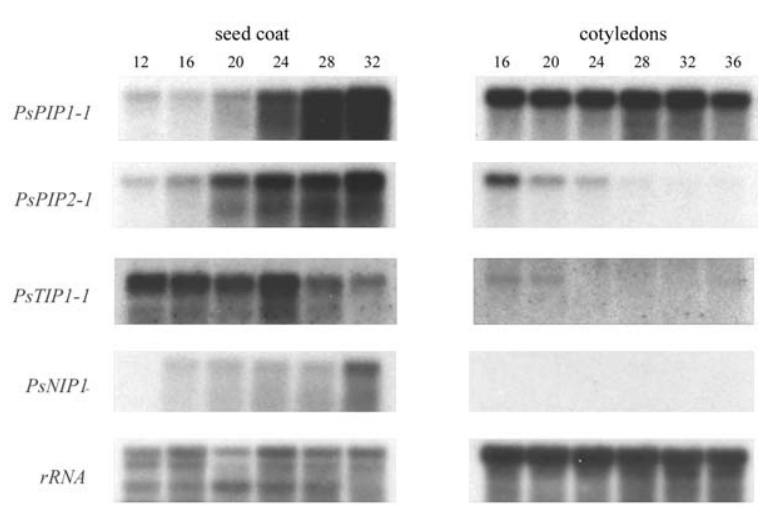

Figure 2. RNA gel blot analysis of seed coat and cotyledons during seed development, with the probes for PsPIP1-1, PsPIP2-1, PsTIP1-1 and PsNIP-1. Tissues were harvested 12-36 days after flowering (d.a.f.).

no cross-hybridization, but it is possible that each probe also hybridized with mRNA of its possible close homologues. This is particularly true of the probes of PSPIP1-1 and PSPIP2-1 because it is likely that in pea, as in Arabidopsis and maize, some members of the PIP1 and PIP2 subfamilies are highly similar isoforms. PsPIP1-1 and PsPIP1-2 may be such isoforms since their PCR fragments showed $83 \%$ identity.

The expression patterns in seed coat and cotyledons during seed development are shown in Figure 2. In cotyledons a high and remarkably constant hybridization signal was observed with the PsPIPI-1 probe, whereas the signals with the probes of PSPIP2-1 and PsTIP1-1 were confined to the earlier stages of development ( $<24$ days after flowering), and transcripts of PSNIP-1 could not be detected at all. In seed coats the hybridization signal for the PSTIPI-1 probe was almost invariable up to 28 days after flowering, and then slightly decreased. By contrast, the signals observed with the probes of PSPIP1-1, PsPIP2-1 and $P S N I P-1$ strongly increased in the final stage of seed development ( $>20$ days after flowering).

In the dry, mature seed transcripts of PSPIPI-1 and/or its close homologues were abundantly present whereas those of PsPIP2-1, PsTIP1-1 -and PSNIP1 were not detected (Figure 3). After imbibition the hybridization signal of the PSPIP1-1 probe in the cotyledons first declined, but increased again after four days of imbibition. Hybridization signals of the PSTIP1-1 probe also reappeared in the cotyledons of the germinating seed, whereas transcripts of PSNIP1 and PSPIP2-1 remained undetectable. In roots of the etiolated pea seedlings high hybridization signals were seen with the probes of PSPIP1-1, PSPIP2-1 and 


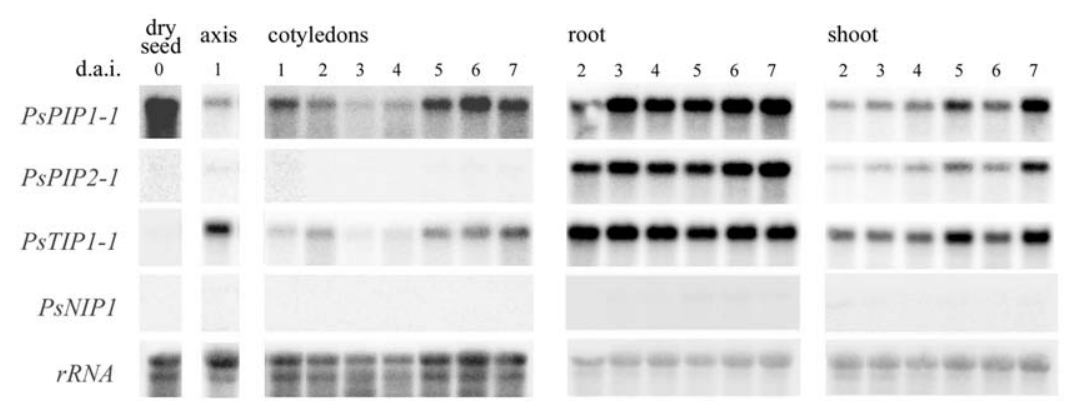

Figure 3. RNA gel blot analysis of dry seeds and of cotyledons, shoots, and roots of etiolated seedlings, using the probes for PsPIP1-1, PsPIP2-1, PsTIP1-1 and PSNIP-1. Tissues were harvested 0-7 days after imbibition (d.a.i.).

PSTIP1-1. The signals were lower in seedling shoots and tended to increase during the 7-day growth period. Transcripts of PSNIP- 1 could not be detected in roots and shoots of the seedlings.

\section{Functional characterization}

The coding sequences of PsPIP1-1, PsPIP2-1, PSTIP1-1, and PSNIP-1 were sub-cloned into a vector designed for the preparation of cRNA required for transient expression in Xenopus oocytes. The osmotic water permeability $\left(P_{\mathrm{f}}\right)$ of oocytes was determined from the initial swelling rate of oocytes after transfer to a hypotonic solution (Figure 4). Expression of PSPIP1-1 in Xenopus oocytes had no effect on the swelling rate of the oocytes, whereas PsPIP2-1 increased $P_{\mathrm{f}}$ about 5 times, from $10.2 \pm 0.7 \mu \mathrm{m} / \mathrm{s}$ to $52.3 \pm 4.2 \mu \mathrm{m} / \mathrm{s}$. After expression of PSTIPI-1 and $P S N I P-1$, the $P_{\mathrm{f}}$ of oocytes slightly but significantly increased to $12.3 \pm 0.7 \mu \mathrm{m} / \mathrm{s}$ and $13.6 \pm 0.9 \mu \mathrm{m} / \mathrm{s}$, respectively.

With the oocyte expression system we also tested the permeability of the four MIPs for urea, glycerol, glycine, and orthophosphate. The permeability of certain aquaporins for glycerol and/or urea is well known, and evidence that GlpF is permeable for glycine has been reported (Heller et al., 1980). We also tested the permeability for phosphate because the cDNAs of two MIP genes from potato have been found to complement a phosphate uptake- deficient yeast mutant (Leggewie et al., 1995). In some experiments a highly significant stimulation of solute uptake was observed. For instance, in one experiment conducted at $\mathrm{pH} 5.5$, the expression of PsPIP1-1 increased the uptake of glycerol and glycine more than three-fold, whereas no stimulation of the uptake of urea and phosphate was observed (not shown). Unfortunately, these results could not be reproduced in further experiments. In fact, we were unable to show any reproducible stimulation in the uptake of urea, glycine, or phosphate after the expression of PSPIP1-1, PSPIP2-1, or PsTIP1-1. Uptake of glycerol by oocytes, however, was consistently enhanced after the expression of PSNIP-1 but not after expression of the other three seed coat MIPs (Figure 5).

\section{Discussion}

\section{Aquaporins and the water relations of developing} seeds

During the early stages of development, the dry matter content of the pea seed (ca. 20\%) is similar to that of the phloem sap (Lewis and Pate, 1973), which demonstrates that the developing seed can obtain sufficient water from the phloem sap stream. The vascular bundle that runs from the funiculus into the seed coat consists largely of phloem elements but it also contains a central xylem strand (van Dongen et al., 2003). With acid fuchsin as a tracer it can easily be shown, however, that water from the xylem sap stream does not enter into developing legume seeds (P. Wolswinkel and J.T. van Dongen, unpublished observations). It is well known that the water relations of developing seeds are largely independent of those in the rest of the plant (Bradford, 1994; Shackel and Turner, 2000), possibly because water is supplied exclusively by the phloem.

At the end of the filling stage, but well before the final desiccation, the dry matter content of the pea seed had increases to ca. $45 \%$. During seed fill, therefore, the phloem sap stream supplies more water than is needed by the seed. The excessive amount of water is probably cycled back into the xylem, but it is not known which cells are involved in this water flux.

Phloem water entering the developing seed has first to be released from the seed coat symplast by crossing 


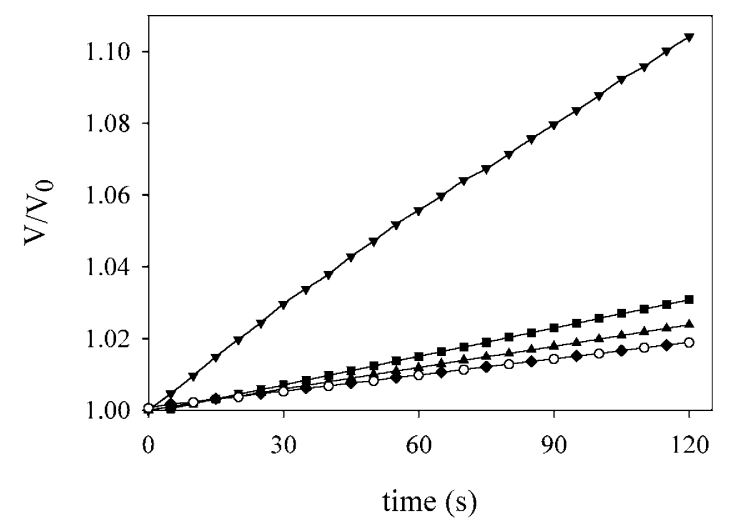

Figure 4. Relative volume change $\left(V / V_{0}\right)$ of oocytes subjected to a swelling assay. Oocytes of Xenopus laevis were injected with water $(\bigcirc)$ or with $25 \mathrm{ng}$ cRNA coding for PsPIP1-1 ( $)$, PsPIP2-1 $(\boldsymbol{\nabla})$, PsTIP1-1 ( $\mathbf{\Delta})$ and PsNIP-1 ( $\mathbf{\square})$. The next day, the vitelline membrane was peeled of and oocytes were placed in $10 \%$ MBS. Volume changes of individual oocytes from four different frogs were monitored during the first two minutes. For each MIP the result obtained with a single, representative oocyte is shown. Mean values of the osmotic water permeability $\left(P_{f}\right.$ in $\left.\mu \mathrm{m} / \mathrm{s}\right)$ were as follows: PsPIP2-1, 52.3 \pm 4.2; PsNIP-1, 13.6 \pm 0.9 ; PsTIP1-1, 12.3 \pm 0.7 ; PsPIP1-1, 9.8 \pm 0.5 ; control (water injected), 10.2 \pm 0.7 .

the plasma membranes of sieve element/companion cell complexes or seed coat parenchyma cells. It seems likely that the aquaporin PsPIP2-1, possibly along with PsPIP1-1 (see below), participates in regulating the transmembrane water movements that are linked to phloem unloading. The possibility that additional aquaporins play a role cannot be excluded because our inventory of aquaporin genes expressed in the pea seed coat is probably not exhaustive.

Translocation of assimilates through the phloem is a hydraulically operated process, but information about the involvement of aquaporins is scanty (Patrick et al., 2001). A study of the distribution of PIP1 and TIP1 homologous proteins in leaves of Brassica napus showed that both types of protein were present in phloem elements of the minor veins but at a much lower level than in parenchyma cells of mesophyll and vascular bundles (Frangne et al., 2001). In phloem sap of barley transcripts of the PIP1 member emip have been detected (Doering-Saad et al., 2002).

\section{Abundant transcripts of a PIP1 homologue in the dry seed}

Inasmuch as PIP2 members expressed in Xenopus oocytes induce a relatively high water permeability they may be regarded as the water channels in plant plasma membranes. The function of PIP1 members is less

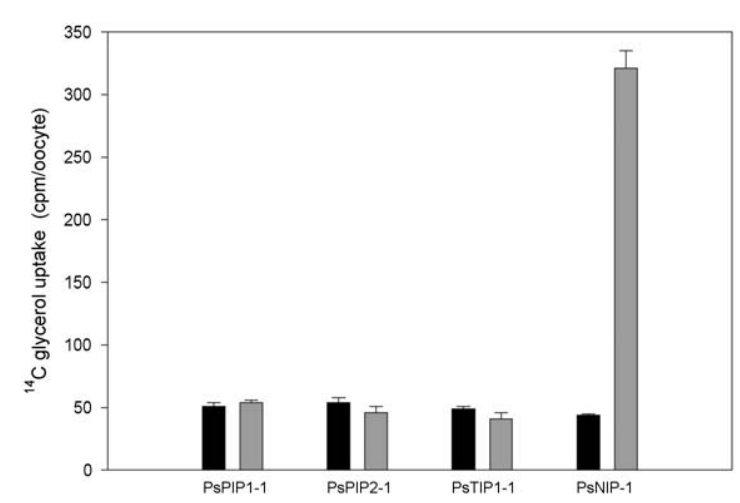

Figure 5. Glycerol uptake by Xenopus oocytes injected with water (black columns) or with 25 ng cRNA coding for PsPIP1-1, PsPIP2-1, PsTIP1-1 and PsNIP-1 (gray columns). The results are from representative experiments, each with 18-24 oocytes. Experiments with PsTIP1-1 and PsNIP-1 were repeated once, that with PsPIP1-1 three times.

clear because, after expression in oocytes, they show a much lower aquaporin activity or no activity at all (Figure 4; Chaumont et al., 2000; Moshelion et al., 2002). Nevertheless, evidence is now accumulating that PIP1 members function in the plant as aquaporins. First, it may be noted that the amino acid residues predicted to border the selectivity filter are the same in PIP1 and PIP2 members (exemplified in Table 1 for PsPIP1-1 and PsPIP2-1). Secondly, recent studies in which the expression of PIP1 members was either impaired or enhanced have provided strong evidence that PIP1 members function in cellular and whole-plant water relations (Siefritz et al., 2002; Martre et al., 2002; Aharon et al., 2003).

Members of PIPI and PIP2 are often co-expressed in plant tissues, but in the dry seed transcripts of PSPIP2-1 could not be detected (Figure 3 ). The absence of PIP2 members in dry seeds has been noted before. Neither the RD28 protein from Arabidopsis nor the mRNA of BnPIPl from Brassica napus was detectable in dry seeds (Daniels et al., 1994; Gao et al., 1999). In dry seeds of radish transcripts of both a PIP2 homologue (PAQ2) and a PIP1 homologue (PAQ1) were undetectable (Suga et al., 2001). We found the mRNA of PSPIP1-1 and/or its possible close homologues to be abundantly present in the dry pea seed (Figure 3). To the best of our knowledge, this is the first demonstration of transcripts of a PIP homologue in a dry seed, which raises the possibility that PIP1 members play a role in the rehydration of the dry seed during imbibition. 
Table 1. Amino acid residues lining the selectivity filter and at Froger's positions $\left(\mathrm{P}_{1}-\mathrm{P}_{5}\right)$ in AQP1 and GlpF and the corresponding residues in several other MIPs. The functional characterization as aquaporin or aquaglyceroporin, and the subgroups to which the plant MIPs belong are indicated. Tryptophan residues corresponding with Trp-48 in GlpF and the residues considered being typical of glycerol transporters (Froger et al., 1998) are printed in bold.

\begin{tabular}{llllllllllll}
\hline Code & Description & \multicolumn{2}{l}{ Selectivity filter } & & $\mathrm{P}_{1}$ & $\mathrm{P}_{2}$ & $\mathrm{P}_{3}$ & $\mathrm{P}_{4}$ & $\mathrm{P}_{5}$ \\
\hline HsAQP1 & human aquaporin & F & R & C & H & T & S & A & F & W \\
CvAQPcic & insect aquaporin & F & R & S & H & T & S & A & Y & W \\
EcAqpZ & bacterial aquaporin & F & R & T & H & A & S & A & F & W \\
PsPIP1-1 & PIP1 & F & R & T & H & E & S & A & F & W \\
PsPIP2-1 & PIP2; aquaporin & F & R & T & H & Q & S & A & F & W \\
NtAQP1 & PIP1; aquaglyceroporin & F & R & T & H & M & S & A & F & W \\
SsAQP1 & PIP1; aquaglyceroporin & F & R & T & H & E & S & A & F & W \\
PsTIP1-1 & TIP1; aquaporin & F & V & A & I & T & S & A & Y & W \\
NtTIPa & TIP4; aquaglyceroporin & H & R & A & I & T & S & A & Y & W \\
GmNod26 & NIP; aquaglyceroporin & W & R & A & V & F & S & A & Y & L \\
PsNIP-1 & NIP; aquaglyceroporin & W & R & A & V & F & S & A & Y & M \\
LjLIMP2 & NIP; aquaglyceroporin & W & R & A & V & F & S & A & Y & L \\
AtNLM1 & NIP; aquaglyceroporin & W & R & A & V & F & S & A & Y & L \\
AtNLM2 & NIP; aquaglyceroporin & W & R & A & V & F & S & A & Y & I \\
PtNIP1;1 & NIP; aquaglyceroporin & W & R & A & V & F & T & A & Y & M \\
EcGlpF & bacterial aquaglyceroporin & W & R & F & G & Y & D & K & P & L \\
ScFPS1 & yeast aquaglyceroporin & W & R & T & N & W & D & R & P & M \\
HsAQP3 & human aquaglyceroporin & F & R & Y & G & Y & D & R & P & I \\
\hline
\end{tabular}

The analyzed sequences with accession numbers and species names are listed: AtNLM1 (O48595) and AtNLM2 (O49406) from Arabidopsis thaliana; CvAQPcic (Q23808) from Cicadella viridis (green cicada); EcAqpZ (P48838) and EcGlpF (P11244) from Escherichia coli; GmNod26 (P08995) from Glycine max (soybean); HsAQP1 (P29972) and HsAQP3 (Q92482) from Homo sapiens; LjLIMP2 (Q9LKJ5) from Lotus japonicus; NtTIPa (Q9XG70) and NtAQP1 (O24662) from Nicotiana tabacum (common tobacco); PsPIP1-1 (P25794), PsPIP2-1 (Q9XGG8), PsTIP1-1 (Q9XGG6) and PsNIP-1 (Q9XGG7) from Pisum sativum (garden pea); PtNIP1;1 (Q940D9) from Pinus taeda (loblolly pine); ScFPS1 (P23900) from Saccharomyces cerevisiae (baker's yeast); SsAQP1 (O65356) from Samanea saman (rain tree).

\section{Some TIP1 members ( $\gamma$ TIPs) are highly expressed in tissues with intensive symplasmic nutrient transfer}

The TIP subfamily can be divided into five subgroups (Johanson et al., 2001; Chaumont et al., 2001), of which the $\alpha-, \gamma$-, and $\delta$-TIPs have been studied most extensively. Vacuoles containing seed-type storage proteins are marked by $\alpha$-TIPs together with $\gamma$ - and/or $\delta$-TIPs, whereas those marked by $\gamma$-TIP alone have characteristics of lytic vacuoles (Jauh et al., 1999). Being the aquaporins of the tonoplast, TIPs are thought to permit a more rapid transcellular water flow by increasing the effective cross-section of the cytoplasm, and to facilitate osmotic adjustment between cytosol and vacuole. It has been proposed that cells with an intensive symplastic transport of assimilates or mineral ions may need an increased capacity for intracellular osmotic equilibration (Barrieu et al., 1998).
The TIP identified in the cDNA library of pea seed coats clearly belongs to the TIP1 subgroup. PSTIP11 and/or its possible close homologues, was expressed in cotyledons, in roots and shoots of seedlings, and at a fairly high level in the seed coat (Figures 2 and 3). The pea seed coat consists of an outer sclerenchyma layer and an inner layer of parenchyma (chlorenchyma, ground parenchyma and branched parenchyma) in which three vascular bundles are embedded. In the stage of seed fill the branched parenchyma has become deteriorated, and the cells of the chlorenchyma/ground parenchyma layer account for some $70-80 \%$ of the tissue volume of the seed coat. These parenchyma cells are likely to be involved in nutrient transfer, as they appear to be symplasmically connected with the sieve elements in the vascular bundles (van Dongen et al., 2003). 
Three other TIP1 members are known that are expressed in roots, stems, and leaves as well as in developing seeds where they localize to tissues involved in nutrient transfer. Thus, $\gamma$ TIPl (=TIP1-1) from Arabidopsis is expressed in the seed coat (Ludevid et al., 1992), ZmTIP1 (= ZmTIP1-1) from maize in pedicel parenchyma cells (see Figure 4 in Barrieu et al., 1998), and MIPFG, a TIP1 member from Norway spruce (Picea abies) in tapetal-like cells around gametophytes and cells of the future seed coat (Hakman and Oliviusson, 2002).

Transcripts of TIP1-members have never been detected in dry seeds (Ludevid et al., 1992; Gao et al., 1999; Suga et al., 2001). In the cotyledons of the pea seed hybridization signals of the PSTIP $1-1$ probe disappeared during seed fill, were undetectable in the dry seed, and reappeared during germination (Figures 2 and 3). Interestingly, the reverse has been found for $\alpha$-TIPs (TIP3 members), which accumulate late in seed maturation and disappear during germination and seedling growth (for references, see Maurel et al., 1997).

\section{An aquaglyceroporin is specifically expressed in the seed coat}

We also identified a NIP that seems to be expressed specifically in the seed coat. NIPs are named after Nodulin-26, an abundant intrinsic protein in the peribacteroid membrane of the soybean nodule, and the most extensively studied member of the subfamily (Dean et al., 1999 and references therein). Nodulin 26 as well as LIMP2 from the legume Lotus japonicus (Guenther and Roberts, 2000) seem to be expressed exclusively in nodules. It may be noted that the peribacteroid membrane of nodule symbiosomes (Udvardi and Day, 1997) and the plasma membrane of the seed coat parenchyma cells have very similar functions, since both membranes have to accommodate the efflux of nutrients from the cytoplasm of the plant cell. We have identified an aquaglyceroporin along with four other MIPs in the seed coat. From a recent study (Wienkoop and Saalbach, 2003) it can be deduced that the peribacteroid membrane of L. japonicus harbors an aquaglyceroporin and at least four other MIPs that can be identified as members of the PIP1, PIP2, TIP1 and TIP2 subgroup, respectively.

PSNIP-1 is another example of a NIP expressed in a reproductive organ. In maize, ZmNIP1-1 and ZmNIP3-1 have the highest expression levels in reproductive tissues (Chaumont et al., 2001). Furthermore, a gene coding for a NIP has been cloned from the pollen of Nicotiana alata (accession number P49173) and another one from rice anthers (Liu et al., 1994), whereas PtNIP1;1 from loblolly pine (Pinus taeda) appeared to be expressed in young, precotyledonary zygotic and somatic embryos, preferentially in the suspensor (Ciavatta et al., 2001).

PsNIP-1 expressed in Xenopus oocytes clearly enhanced the permeability of the oocyte membrane for water and glycerol (Figures 4 and 5). Five other NIPs have been also characterized as channels for water and glycerol (Nodulin-26, Dean et al., 1999; LIMP2, Guenther and Roberts, 2000; AtNLM1 and AtNLM2, Weig and Jakob, 2000; PtNIP1;1, Ciavatta et al., 2001). In GlpF glycerol is transported through an amphipathic channel. The selectivity filter in this channel is lined by four residues, Trp-48, Phe-200, Arg-206, and Gly-199, which are crucial for the selective transport of glycerol (Fu et al., 2000; Sui et al., 2001). Alignment of several glycerol-transporting MIPs showed that Trp-48 in GlpF is conserved in Fsp1, the glycerol facilitator from Saccharomyces cerevisiae, whereas it is replaced by phenylalanine in mammalian aquaglyceroporins such as AQP3. The tryptophan side-chain is also present in PsNIP-1 and all other members of the NIP subfamily that have been experimentally characterized as aquaglyceroporins (Table 1). The importance of the tryptophan residue for glycerol permeation was recently demonstrated for LIMP2 (Wallace et al., 2002). Some NIPs, however, do not contain this tryptophan residue (not shown), and their permeation characteristics have yet to be determined.

Froger et al. (1998) pinpointed five positions $\left(\mathrm{P}_{1}-\right.$ $\mathrm{P}_{5}$ ) to distinguish between aquaporins and glycerol facilitators. The residues at $\mathrm{P}_{4} \mathrm{P}_{5}$ have been found determining factors for the selectivity of water and glycerol transport in the insect aquaporin AQPcic (Lagrée et al., 1999), but not in the aquaporin AqpZ and the glycerol facilitator GlpF from E. coli (Borgnia and Agre, 2001). Neither do the amino acid residues at $\mathrm{P}_{2}$ and $\mathrm{P}_{3}$ bear general relevance for the selective transport of glycerol and water because all NIPs experimentally characterized as aquaglyceroporins have a serine (or threonine) and alanine at these positions, which has been considered typical of aquaporins. Note that all glycerol-transporting NIPs have amino acid residues at only two out of the five positions $\left(\mathrm{P}_{1}\right.$ and $\mathrm{P}_{5}$ ) that Froger et al. (1998) regarded as typical of glycerol facilitators (Table 1). 
Glycerol transport has been also reported for PIP1 members from tobacco (NtAQP1) and Samanea saman (SsAQP1) and for a TIP4 member from tobacco (Biela et al., 1999; Gerbeau et al. 1999; Moshelion et al., 2002). This is quite remarkable because in these three MIPs all residues at Froger's positions are typical of aquaporins and, more importantly, the residues predicted to line the selectivity filter in NtAQP1 and SsAQP1 are identical to those in the orthodox PIP2 aquaporins (exemplified in Table 1 for PsPIP2-1).

\section{Solute transport by plant MIPs?}

On the basis of circumstantial evidence it has been suggested repeatedly that plant MIPs may also function as transport channels for solutes. Nodulin 26 has been proposed as a transport channel for malate, which is the major organic nutrient for bacteroids in root nodules (Ouyang et al., 1991). Furthermore, some PIP1 members have been suggested to function as solute transporters. Expression patterns of TRG31 promoter::GUS constructs in transgenic tobacco lead to the idea that TRG-31 (which is identical to PsPIP1-1) could be involved in the transport of assimilates (Jones and Mullet, 1995), and more recently it was suggested that the ripening-associated membrane protein from tomato, TRAMP, which also belongs to the PIP1 group, might be involved in the transport of sugars and/or organic acids (Chen et al., 2001). Finally, the complementation of yeast mutants deficient in phosphate uptake by PIPs from Solanum tuberosum hinted at a possible permeability for phosphate (Leggewie et al., 1995).

From our own experiments and those reported by others (Opperman et al., 1994; Chaumont et al., 2000) it should be concluded, however, that plant MIPs expressed in Xenopus oocytes have shown no permeability for any of a great diversity of solutes including sugars, amino acids and ions. The transport of glycerol by certain plant MIPs seems now very well documented (see above), and transport of urea by a TIP4 member (Gerbeau et al., 1999) and of boric acid by a PIP1 homologue (Dordas et al., 2000) has been reported. But, to the best of our knowledge, there is at present no direct evidence of a plant MIP that serves as a transport channel for any other solute.

\section{Acknowledgements}

Oocytes were kindly provided by Olivier Destrée and Kees Koster (Hubrecht Laboratory, Utrecht), Maarten
Terlouw generously supplied the software for determining oocyte swelling rates, and Erik Jan Kamsteeg and Peter Deen (University of Nijmegen) introduced J.T.v.D. to the techniques of heterologous expression in Xenopus oocytes. Preliminary oocyte experiments were carried out in our lab by Gerco van Eijkenhorst.

\section{References}

Agre, P., Bonhivers, M. and Borgnia, M.J. 1998. The aquaporins, blueprints for cellular plumbing systems. J. Biol. Chem. 273: 14659-14662.

Aharon, R., Shahak, Y., Wininger, S., Bendov, R., Kapulnik, Y. and Galili, G. 2003. Overexpression of a plasma membrane aquaporin in transgenic tobacco improves plant vigor under favorable growth conditions but not under drought or salt stress. Plant Cell 15: 439-447.

Altschul, S.F., Madden, T.L., Schäffer, A.A., Zhang, J., Zhang, Z., Miller, W. and Lipman DJ. 1997. Gapped BLAST and PSIBLAST: a new generation of protein database search programs. Nucl. Acids Res. 25: 3389-3402.

Barrieu, F., Chaumont, F. and Chrispeels, M.J. 1998. High expression of the tonoplast aquaporin ZmTIP1 in epidermal and conducting tissues of maize. Plant Physiol. 117: 1153-1163.

Biela, A., Grote, K., Otto, B., Hoth, S., Hedrich, R. and Kaldenhoff, R. 1999. The Nicotiana tabacum plasma membrane aquaporin NtAQP1 is mercury-insensitive and permeable for glycerol. Plant J. 18: 565-570.

Borgnia, M.J. and Agre, P. 2001. Reconstitution and functional comparison of purified GlpF and AqpZ, the glycerol and water channels from Escherichia coli. Proc. Natl. Acad. Sci. USA 98: 2888-2893.

Borstlap, A.C. 2002. Early diversification of plant aquaporins. Trends Plant Sci. 7: 529-530.

Bradford, K.J. 1994. Water stress and the water relations of seed development: a critical review. Crop Sci. 34: 1-11.

Chaumont, F., Barrieu, F., Jung, R. and Chrispeels, M.J. 2000. Plasma membrane intrinsic proteins from maize cluster in two sequence subgroups with differential aquaporin activity. Plant Physiol. 122: 1025-1034.

Chaumont, F., Barrieu, F., Wojcik, E., Chrispeels, M.J. and Jung, R. 2001. Aquaporins constitute a large and highly divergent protein family in maize. Plant Physiol. 125: 1206-1215.

Chen, G., Wilson, I.D., Kim, S.H. and Grierson, D. 2001. Inhibiting expression of a tomato ripening-associated membrane protein increases organic acids and reduces sugar levels of fruit. Planta 212: 799-807.

Ciavatta, V.T., Morillon, R., Pullman, G.S., Chrispeels, M.J. and Cairney, J. 2001. An aquaglyceroporin is abundantly expressed early in the development of the suspensor and the embryo proper of loblolly pine. Plant Physiol. 127: 1556-1567.

Corpet, F. 1988. Multiple sequence alignment with hierarchical clustering. Nucl. Acids Res. 16: 10881-10890.

Daniels, M.J., Mirkov, T.E. and Chrispeels, M.J. 1994. The plasma membrane of Arabidopsis thaliana contains a mercuryinsensitive aquaporin that is a homolog of the tonoplast water channel protein TIP. Plant Physiol. 106: 1325-1333.

Dean, R.M., Rivers, R.L., Zeidel, M.L. and Roberts, D.M. 1999. Purification and functional reconstitution of soybean nodulin 26. An aquaporin with water and glycerol transport properties. Biochemistry 38: 347-353. 
de Jong, A., Koerselman-Kooij, J.W., Schuurmans, J.A.M.J. and Borstlap, A.C. 1996. Characterization of the uptake of sucrose and glucose by isolated seed coat halves of developing pea seeds. Evidence that a sugar facilitator with diffusional kinetics is involved in seed coat unloading. Planta 199: 486-492.

de Jong, A., Koerselman-Kooij, J.W. Schuurmans, J.A.M.J. and Borstlap, A.C. 1997. The mechanism of amino acid efflux from seed coats of developing pea seeds as revealed by uptake experiments. Plant Physiol. 114: 731-736.

de Jong, A. and Wolswinkel, P. 1995. Differences in release of endogenous sugars and amino acids from attached and detached seed coats of developing pea seeds. Physiol. Plant. 94: 78-86.

Doering-Saad, C., Newbury, H.J., Bale, J.S. and Pritchard, J. 2002. Use of aphid stylectomy and RT-PCR for the detection of transporter mRNAs in sieve elements. J. Exp. Bot. 53: 631-637.

Dordas, C., Chrispeels, M.J. and Brown, P.H. 2000. Permeability and channel-mediated transport of boric acid across membrane vesicles isolated from squash roots. Plant Physiol. 124: 13491361.

Dumont, J.N. 1972. Oogenesis in Xenopus laevis. J. Morphol. 136: $153-180$.

Feinberg, A.P. and Vogelstein, G. 1983. A technique for radiolabelling DNA restriction fragments to high specific activity. Anal. Biochem. 132: 6-13.

Fisher, D.B. 2000. Long-distance transport. In: B.B. Buchanan, W. Gruissem and R.L. Jones (Eds.) Biochemistry \& Molecular Biology of Plants, American Society of Plant Physiologists, Rockville, MD, pp. 730-784.

Frangne, N., Maeshima, M., Schäffner, A.R., Mandel, T., Martinoia, E. and Bonnemain, J.-L. 2001. Expression and distribution of a vacuolar aquaporin in young and mature leaf tissues of Brassica napus in relation to water fluxes. Planta 212: 270-278.

Froger, A., Tallur, B., Thomas, D. and Delamarche, C. 1998. Prediction of functional residues in water channels and related proteins. Prot. Sci. 7: 1458-1468.

Fu, D., Libson, A., Miercke, L.J.W., Weitzman, C., Nollert, P., Krucinski, J. and Stroud, R.M. 2000. Structure of a glycerolconducting channel and the basis for its selectivity. Science 290: 481-486.

Gao, Y.-P., Young, L., Bonham-Smith, P. and Gusta, L.V. 1999. Characterization and expression of plasma and tonoplast membrane aquaporins in primed seed of Brassica napus during germination under stress conditions. Plant Mol. Biol. 40: 635-644.

Gerbeau, P., Güçlü, J., Ripoche, P. and Maurel, C. 1999. Aquaporin Nt-TIPa can account for the high permeability of tobacco cell vacuolar membrane to small neutral solutes. Plant J. 18: 577587.

Guenther, J.F. and Roberts, D.M. 2000. Water-selective and multifunctional aquaporins from Lotus japonicus nodules. Planta 210: 741-748.

Guerrero, F.D. and Crossland, L. 1993. Tissue-specific expression of a plant turgor-responsive gene with amino acid sequence homology to transport-facilitating proteins. Plant Mol. Biol. 21: 929-935.

Guerrero, F.D., Jones, J.T. and Mullet, J.E. 1990. Turgor-responsive gene transcription and RNA levels increase rapidly when pea shoots are wilted. Sequence and expression of three inducible genes. Plant Mol. Biol. 15: 11-26.

Hakman, I. and Oliviusson, P. 2002. High expression of putative aquaporin genes in cells with transporting and nutritive functions during seed development in Norway spruce (Picea abies). J. Exp. Bot. 53: 639-649.
Heller, K.B., Lin, E.C.C. and Wilson, T.H. 1980. Substrate specificity and transport properties of the glycerol facilitator of Escherichia coli. J. Bacteriol. 144: 274-278.

Heymann, J.B. and Engel, A. 1999. Aquaporins: phylogeny, structure, and physiology of water channels. News Physiol. Sci. 14: 187-193.

Ikeda, M., Beitz, E., Kozono, D., Guggino, W.B., Agre, P. and Yasui, M. 2002. Characterization of aquaporin-6 as a nitrate channel in mammalian cells. Requirement of pore-lining residue threonine 63. J. Biol. Chem. 277: 39873-39879.

Jauh, G.-Y., Phillips, T.E. and Rogers, J.C. 1999. Tonoplast intrinsic protein isoforms as markers of vacuolar functions. Plant Cell 11: 1867-1882.

Johanson, U., Karlsson, M., Johansson, I., Gustavsson, S., Sjövall, S., Fraysse, L., Weig, A.R. and Kjellbom, P. 2001. The complete set of genes encoding major intrinsic proteins in Arabidopsis provides a frame work for a new nomenclature for major intrinsic proteins in plants. Plant Physiol. 126: 1358-1369.

Jones, J.T. and Mullet, J.E. 1995. Developmental expression of a turgor-responsive gene that encodes an intrinsic membrane protein. Plant Mol. Biol. 28: 983-996.

Krieg, P.A. and Melton, D.A. 1984. Functional messenger RNAs are produced by SP6 in vitro transcription of cloned cDNAs. Nucl. Acids Res. 12: 7057-7070.

Lagrée, V., Froger, A., Deschamps, S., Hubert, J.-F., Delamarche, C., Bonnec, G., Thomas, D., Gouranton, J. and Pellerin, I. 1999. Switch from an aquaporin to a glycerol channel by two amino acid substitutions. J. Biol. Chem. 274: 6817-6819.

Leggewie, G., Willmitzer, L. and Riesmeier, J.W. 1995. Molecular approaches towards the isolation and characterisation of plant phosphate transporters. 10th International Workshop on Plant Membrane Biology, 6-11 August 1995, Regensburg, p. R32.

Lewis, O.A.M. and Pate, J.S. 1973. The significance of transpirationally derived nitrogen in protein synthesis in fruiting plants of pea (Pisum sativum L.). J. Exp. Bot. 24: 596-606.

Liu, Q., Umeda, M. and Uchimiya, H. 1994. Isolation and expression analysis of two rice genes encoding the major intrinsic protein. Plant Mol. Biol. 26: 2003-2007.

Logemann, J., Schell, J. and Willmitzer, L. 1987. Improved method for the isolation of RNA from plant tissue. Anal. Biochem. 163: 16-20.

Ludevid, D., Höfte, H., Himelblau, E. and Chrispeels, M.J. 1992. The expression pattern of the tonoplast intrinsic protein $\gamma$-TIP in Arabidopsis thaliana is correlated with cell enlargement. Plant Physiol. 100: 1633-1639.

Martre, P., Morillon, R., Barrieu, F., North, G.B., Nobel, P.S. and Chrispeels, M.J. 2002. Plasma membrane aquaporins play a significant role during recovery from water deficit. Plant Physiol. 130: 2101-2110.

Maurel, C., Chrispeels, M., Lurin, C., Tacnet, F., Geelen, D., Ripoche, P. and Guern, J. 1997. Function and regulation of seed aquaporins. J. Exp. Bot. 48: 421-430.

Moshelion, M., Becker, D., Biela, A., Uehlein, N., Hedrich, R., Otto, B., Levi, H., Moran, N. and Kaldenhoff, R. 2002. Plasma membrane aquaporins in the motor cells of Samanea saman: diurnal and circadian regulation. Plant Cell 14: 727-739.

Münch, E. 1930. Die Stoffbewegungen in der Pflanze. Gustav Fischer, Jena.

Opperman, C., Taylor, C.G. and Conkling, M.A. 1994. Rootknot nematode-directed expression of a plant root-specific gene. Science 263: 221-223.

Ouyang, L.J., Whelan, J., Weaver, C.D., Roberts, D.M. and Day, D.A. 1991. Protein phosphorylation stimulates the rate of malate 
uptake across the peribacteroid membrane of soybean nodules. FEBS Lett. 293: 188-190.

Patrick, J.W. and Offler, C.E. 2001. Compartmentation of transport and transfer events in developing seeds. J. Exp. Bot. 52: 551564.

Patrick, J.W., Zhang, W., Tyerman, S.D., Offler, C.E. and Walker, N.A. 2001. Role of membrane transport in phloem translocation of assimilates and water. Aust. J. Plant Physiol. 28: 695-707.

Sambrook, J., Fritsch, E.F. and Maniatis, T. 1989. Molecular Cloning: A Laboratory Manual, 2nd ed. Cold Spring Harbor Laboratory Press, Plainview, NY.

Shackel, K.A. and Turner, N.C. 2000. Seed coat cell turgor in chickpea is independent of changes in plant and pod water potential. J. Exp. Bot. 51: 895-900.

Siefritz, F., Tyree, M., Lovisolo, C., Schubert, A. and Kaldenhoff, R. 2002. PIP1 plasma membrane aquaporins in tobacco: from cellular effects to function in plants. Plant Cell 14: 869-876.

Silberberg, S.D. and Magleby, K.L. 1997. Voltage-induced slow activation and deactivation of mechanosensitive channels in Xenopus oocytes. J. Physiol. (London) 505: 551-569.

Suga, S., Imagawa, S. and Maeshima, M. 2001. Specificity of the accumulation of mRNAs and protein of the plasma membrane and tonoplast aquaporins in radish organs. Planta 212: 294-304.

Sui, H., Han, B.-G., Lee, J.K., Walian, P. and Jap, B.K. 2001. Structural basis of water-specific transport through the AQP1 water channel. Nature 414: 872-878.

Tsukaguchi, H., Shayakul, C., Berger, U.V., Mackenzie, B., Devidas, S., Guggino, W.B., van Hoek, A.N. and Hediger, M.A. 1998.
Molecular characterization of a broad selectivity neutral solute channel. J. Biol. Chem. 273: 24737-24743.

Udvardi, M.K. and Day, D.A. 1997. Metabolite transport across symbiotic membranes of legume nodules. Annu. Rev. Plant Physiol. Plant Mol. Biol. 48: 493-523.

van Dongen, J.T., Ammerlaan, A.M.H., Wouterlood, M., van Aelst, A.C. and Borstlap, A.C. 2003. Structure of the developing pea seed coat and the post-phloem transport pathway of nutrients. Ann. Bot. 91: 729-737.

van Dongen, J.T. and Borstlap, A.C. 2003. Aquaporins: structure, function and phylogenetic analysis. In: A.R. Hemsley and I. Poole (Eds.) The Evolution of Plant Physiology, Academic Press, London, pp. 109-120.

van Dongen, J.T., Laan, R.G.W., Wouterlood, M. and Borstlap, A.C. 2001. Electrodiffusional uptake of organic cations by pea seed coats. Further evidence for poorly selective pores in the plasma membrane of seed coat parenchyma cells. Plant Physiol. 126: 1688-1697.

Wallace, I.S., Wills, D.M., Guenther, J.F. and Roberts, D.M. 2002. Functional selectivity for glycerol of the nodulin 26 subfamily of plant membrane intrinsic proteins. FEBS Lett. 523: 109-112.

Weig, A.R. and Jakob, C. 2000. Functional identification of the glycerol permease activity of Arabidopsis thaliana NLM1 and NLM2 proteins by heterologous expression in Saccharomyces cerevisiae. FEBS Lett. 481: 293-298.

Wienkoop, S. and Saalbach, G. 2003. Proteome analysis. Novel proteins identified at the peribacteroid membrane from Lotus japonicus root nodules. Plant Physiol. 131: 1080-1090. 